\title{
Vision-based Navigation in Service Robotics
}

\author{
G.Garibotto, M.Ilic, S.Masciangelo \\ TELEROBOT \\ via Hermada 6, Genova, Italy
}

\begin{abstract}
The paper describes an integrated mobile robot which has been designed to perform autonomous mission in service robotics. Computer Vision represents the main sensory system for autonomous navigation by artificial landmark recognition and $3 \mathrm{D}$ location. The orientation control through vanishing point analysis is performed by Kalman filtering. The system is proved to successfully work in a crowded scenario, it requires minimal impact on the environment, with a friendly user interface.
\end{abstract}

\section{Introduction}

Service Robotics is an emerging field of applications aimed to provide the necessary flexibility in performing repetitive tasks, as light material transportations (mail, documents, medicines, clinical data), and for heavy transportations (warehouse management and material handling). There are prospects [1] of wide expansion in various different fields, from hostile environments (nuclear, chemical) in surveillance and patrolling, entertainment, up to future domestic applications as cleaning and assistance.

A basic requirements in all these applications is autonomous navigation with capabilities to adapt to the different operating conditions. In order to demonstrate the maturity of vision-based navigation technology, also using low-cost PC-based processing platform, we have decided to realize a prototype of a mobile robot, named SAM (Autonomous Mobile System), suitable to address a sufficiently representative class of autonomous navigation and transport tasks, in an indoor environment, in presence of people.

Moreover, we have also experienced a novel application of service robotics, as a museum guide. The robot, equipped with a sound/voice generation system, was installed at Palazzo Ducale, Genova, an historical building where temporal expositions are periodically held. The robot was able to navigate between points of interests where it could stop and generate a vocal message or a sound. Moreover other messages were generated according to the particular navigation condition; for instance, the presence of people along the navigation path was managed by the by means of a proper message or sound. This paper does not include a description of the vocal interface, which is available in [6], but is mainly focused on the platform navigation capabilities. In the next section an overview of the prototype is provided, then, some technical aspects of the prototype are described in greater detail with particular reference to vision based global positioning subsystem. Finally some experimental results are referred in terms of reliability and easy of use of the system. 


\section{Description of the mobile robot SAM}

The logic architecture is decomposed in almost independent layers of competencies, each one in charge of a single, well defined task, such as obstacle avoidance or global position maintenance. The obstacle avoidance strategy is reflexive, that is the trajectory is heuristically determined on the basis of sensor readings rather than accurately planned starting from a reconstructed local environmental map. The suboptimality of the obtained trajectory is largely compensated by the fast response time which allows to navigate safely at an acceptable speed also in presence of cluttered environments. Moreover map making and planning capabilities are limited as far as possible in order to spare computational power and software development costs.
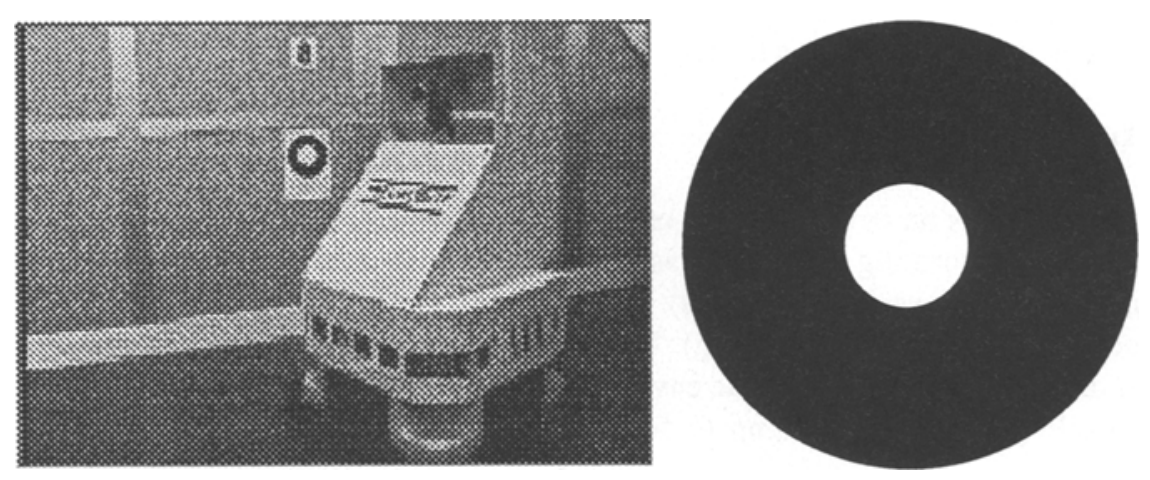

Figure 1: The mobile robot SAM

Figure 2: The artificial landmark

The hardware solution is based on a PC platform as the main computational infrastructure to reduce costs, minimise the development time and take advantage of the wide choice among a great variety of add-on boards which can be integrated to improve the system functionality.

\section{Computer Vision for Autonomous Navigation}

\subsection{High level planning and pose computation}

The navigation system needs a periodic position and orientation estimation coming from an external sensor in order to reset drifts of odometry.

This is provided through a Vision system able to detect and recognise navigation landmarks placed in known position along the robot routes [4], and to recover the robot position and orientation with respect to them. A navigation landmark may an object already present in the environment (natural landmarks) or a feature placed on purpose along the robot routes (artificial landmarks).

In our approach, each artificial landmark consists of a black annulus on a white background, visible in Fig.2. The technique that allows to recover the 3D position 
and attitude of the camera from a single image is known as model based perspective inversion [2].

Although the use of artificial landmark is a reliable way to solve the problem of selfpositioning, it requires an intervention on the environment, which should be minimized, as far as possible. Anyway, when moving along hallways, it is possible to recover the robot absolute orientation from the analysis of the vanishing points. They are natural landmarks, since they are already present in the world and may be extracted reliably in indoor environments, where the contours of visible surfaces are mainly formed by either parallel or mutually orthogonal straight lines. The vanishing point is invariant to camera translation movements, therefore its tracking along an image sequence can give an estimate of the camera orientation [3].

Which of the two Vision algorithms is invoked is specified in the global map and therefore depends on the robot current position. The global map is not a complete 3D map but rather an abstract description based on points of interest located in strategic positions for the navigation.

Neighbouring points of interest are connected by vectors, whose module represents the length of the path and the vector orientation is the robot's heading direction. A valid navigation plan is a sequence of points of interest, or subgoals, to be reached.

When the robot reckons to be on a point of interest, it can take either of the following actions:

- Stop and verify its position by observing the relative artificial landmark or vanishing point (this is specified in an attribute of the point of interest), reset odometric errors and turn towards the following subgoal;

- Execute a motion command such as an absolute turn.

Landmarks differ each other for the diameter of the inner circle. This parameter, that can be measured very accurately on the image plane, is a perspective invariant, i.e., does not change with respect to the viewpoint. That property, together with the perspective inversion, allows to determine the absolute location of the robot in the global map and to replan the mission, that is the next path segment towards the given final goal.

The same search strategy allows the robot to start the mission from any point in which at least one landmark is visible, without any a-priori information on the starting position.

\subsection{Heading control from visually detected vanishing point direction}

In order to improve the reliability of visual navigation a continuous visual control algorithm which employs together vision and odometry has been developed and integrated into the navigation system. The module controls the robot's heading angle by exploiting again the vanishing point generated by the 3D worls structure typical of indoor environment. In fact horizontal parallel lines generate two bundles, each one meeting the horizon line at infinity in a vanishing point, as shown in Fig. 3.b. By extension, the direction of the straight line that links the vanishing point to the camera pinhole is also called vanishing point. Thus the heading of the vehicle can be recovered from one of the two vanishing points, by adding the fixed angle between 
the forward vehicle direction, known with respect to the odometry coordinate system, and the projection of the camera optical axis onto the horizon, estimated by calibration.

In order to extract 3D geometric information, as the orientation of a vanishing point, from the 2D features of an image, it is necessary to compute, off-line, the camera intrinsic parameters as well as its orientation and position with respect both to the vehicle odometry coordinate system and the horizontal navigation plane. All these parameters are computed jointly by moving the vehicle in different positions and using the corresponding odometric information.

\subsection{Sensor fusion through Kalman filtering}

The integration with the odometry has been carried out through Kalman filtering, to deal with different confidence values (variance data) of the input measures, which are acquired at different time scales, according to their computational requirements. The process equation for the vehicle heading angle $\theta_{i}$ at time $t_{i}$ for $i>0$ may be written as

$$
\theta_{i}=\theta_{i-1}+\Delta \theta_{\text {odo }_{i-1}}+w_{i-1} \text { where }\left\{\begin{array}{c}
w_{i}=\Delta \theta_{i}-\Delta \theta_{\text {odo }_{i}} \\
\Delta \theta_{i}=\theta_{i+1}-\theta_{i} \\
\Delta \theta_{\text {odo }_{i}}=\theta_{\text {odo }_{i+1}}-\theta_{\text {odo }_{i}}
\end{array}\right.
$$

and $\theta_{\mathrm{odo}_{i}}$ is the value of the odometry heading angle register on the vehicle as read at time $t_{i}$.

While performing explicit Kalman filter steps at vision measurement rate only, this approach exploits the odometry system embedded in the platform controller, according to the block diagram of Fig. 3.a, which polls encoders at higher rate. It would be interesting to work out a statistical model also for the odometry computation process in order to better integrate it in the data fusion schema provided by the Kalman filter. More details on the Kalman filter implementation can be found in [7]

Experiments on the performance and use of the fusion with the odometry of the heading angle measurements from vanishing point detection have been carried out on the SAM mobile system. Commands were issued to make the vehicle navigate straight ahead for $5000 \mathrm{~mm}$ at $250 \mathrm{~mm} / \mathrm{s}$ constant speed.

In order to emphasise the effect of vision measurements updates with respect to a poor odometry performance, the path was suitably arranged to make the vehicle navigate with one wheel on the floor while the other was slipping on a thick carpet. Without vision this resulted in a great error of the final lateral displacement, about $0.5 \mathrm{~m}$, and of heading, about 15 degrees, which was nonetheless recovered as soon as the vision-based heading measures have been introduced. In order to allow a motion feedback control loop based not only on wheel encoder readings, but also on the current odometry heading register, a high-level control loop was added to generate, while advancing, a corrective rotational velocity proportional to the heading angle error as reported by the odometry heading register. 

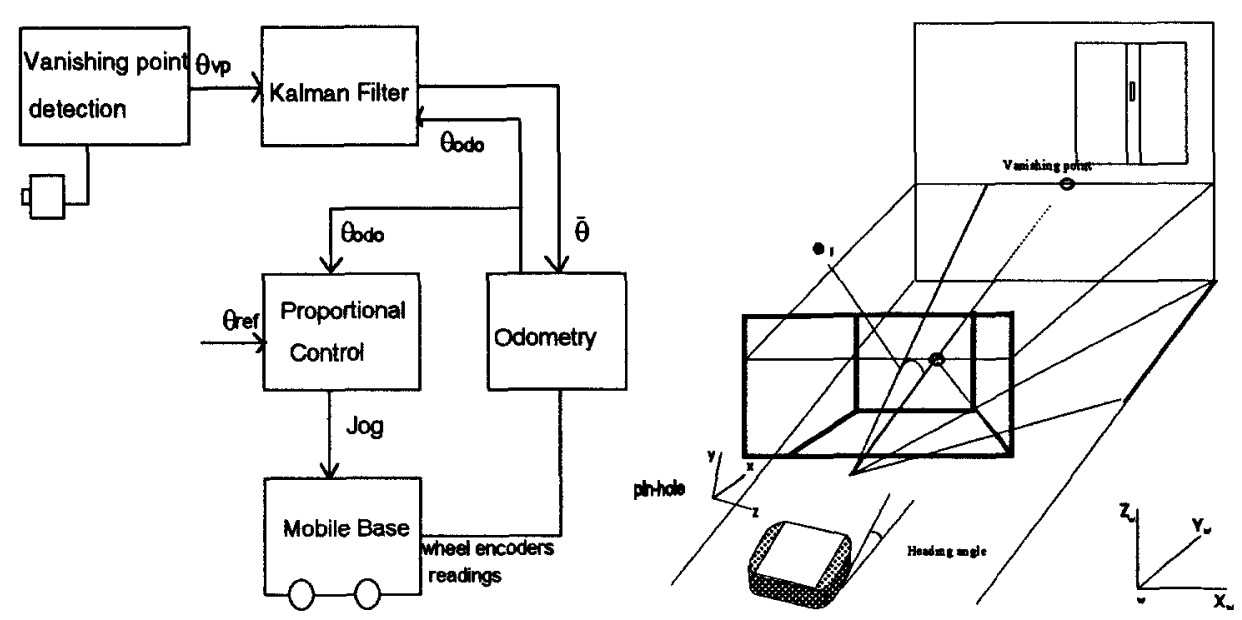

Figure 3: a) Schema of the vehicle direction feedback control system. b).The vanishing point of the scene and its mapping onto the image plane. The vehicle Heading angle is also shown.

The rate of this control was four times higher than the vision-based heading measurement procedure, whose average execution time was $0.7 \mathrm{~s}$ for a scene showing about 350 segments, on a PC486 33MHz. The final lateral displacement error was reduced to $0.25 \mathrm{~m}$ and the heading error to $1.5^{\circ}$. Even if the lateral displacement was not explicitly controlled, the vehicle heading feedback loop kept the vehicle from drifting aside too much, showing once again that the most important navigation control variable is heading.

\section{Experimental results}

It is important to evaluate the system as a whole more than the single modules. Experiments consisted in making the robot move on a fixed route first in our office environment, usually full of people wandering around. Later the robot has been equipped with a Soundblaster ${ }^{\mathfrak{C}}$ board for sound generation and a radio modem providing a link to an host computer at a remote control station. The remote computer is able to select the most appropriate sound or voice file according to the robot position or the navigation status (presence of obstacles, landmark search, and so on) as communicated by the robot navigation system.

The robot in such a configuration was installed in an historical building during the Christmas ' 93 exhibitions, as reported in Table 1. Environments were not artificially structured, apart from the application of few circular landmarks $\left(20 \mathrm{~cm}^{2}\right)$ on the walls. General public, mainly composed by children, was not prepared to any interaction with the robot, therefore it was not possible to foresee the reaction and the behaviour in presence of it. 
The results were very encouraging and showed that the obstacle avoidance system is quite reliable for the detection of people and that the vision system was able to work robustly in every light condition. Problems came from the excessive number of people surrounding the robot, who occluded the camera field of view and from the very bad vehicle odometry, depending on the floor conditions, that caused occasionally mission aborts in between two points of self-positioning.

\begin{tabular}{|l|l|}
\hline Navigation map & $\begin{array}{l}\text { a corridor } 30 \mathrm{~m} \text { long and } 7 \mathrm{~m} \text { wide, plus a large area 20 } \\
\text { X } 15 \mathrm{~m}\end{array}$ \\
\hline Artificial landmarks & 5 \\
\hline Function & $\begin{array}{l}\text { attraction and entertainment, explanation about the } \\
\text { exhibitions by vocal interface }\end{array}$ \\
\hline Duration & four days including rehearsals, about 25 hours \\
\hline Public & high density of people, great interest among children \\
\hline Cruise speed & $60 \mathrm{~cm} / \mathrm{s}$ \\
\hline Anomalies & $\begin{array}{l}\text { a user intervention every 10 min in average due to: } \\
\text { landmark occlusion for the presence of people (70 \%), } \\
\text { loss of orientation because of slippage on irregular floor } \\
\text { patches (301\%). }\end{array}$ \\
\hline
\end{tabular}

Table 1: The main characteristics of the experiments carried out at Palazzo Ducale (Genova) during Christmas exhibitions 1993.

\section{References}

[1] J.Engelberger, "Robotics in Service", London, Kogan Page Ltd, 1989.

[2] M. Ferri, F. Mangili, G. Viano, "Projective Pose Estimation and Quadratic Primitives in Monocular Computer Vision", CVGIP: Image Understanding,vol. 58, N. 1, July 1993.

[3] Straforini, C. Coelho and M. Campani, "Extraction of vanishing points from images of indoor and outdoor scenes", Image and vision computing, vol. 11, no 2, March 1993.

[4] G.B. Garibotto and S. Masciangelo, "3D Computer Vision for Navigation/Control of Mobile Robots", in Machine Perception, AGARD Lecture Series 185, 1992.

[5] X. Lebegue and J.K.Aggarwal, "Extraction and Interpretation of Semantically Significant Line Segment for a Mobile Robot ", Proc. 1992 International Conference on Robotics and Automation, Nice, France, May 1992.

[6] G. B. Garibotto, S. Masciangelo, M Ilic, A Camurri, G.Vercellli, "A Mobile Robotic Museum Guide Demonstrating Intelligent Interaction with the Environment and the Public", ISATA'94, Aachen (Germany), October 1994.

[7] G.B. Garibotto, M. Ilic, S. Masciangelo, "Multisensor Integration for Autonomous Mobile Robotics", Proc. of the Workshop of the Italian Association of Artificial Intelligence, Parma, Sept. 1994 